\title{
ADUBAÇÕES NITROGENADA E POTÁSSICA NO SORGO BIOMASSA -PRODUTIVIDADE E QUALIDADE DE FIBRA
}

\author{
FLÁVIA CRISTINA DOS SANTOS ${ }^{1}$, MANOEL RICARDO DE ALBUQUERQUE FILHO ${ }^{1}$, \\ ÁlVARO VILELA DE RESENDE ${ }^{1}$, ANTÔNIO CARLOS DE OLIVEIRA ${ }^{1}$, TALITA CAMARGOS \\ GOMES $^{2}$ e MARCIELE SILVA OLIVEIRA ${ }^{3}$
}

\author{
${ }^{1}$ Embrapa Milho e Sorgo, Sete Lagoas, MG, Brasil, flavia.santos@embrapa.br, manoel.ricardo@embrapa.br, \\ alvaro.resende@embrapa.br,antoniocarlos.oliveira@embrapa.br \\ ${ }^{2}$ Universidade Federal de São João del-Rei, Sete Lagoas, MG, Brasil,tgcamargos@yahoo.com.br \\ ${ }^{3}$ Unifemm, Sete Lagoas, MG, Brasil, marcieleol@yahoo.com.br
}

Revista Brasileira de Milho e Sorgo, v.13, n.1, p. 1-13, 2014

\begin{abstract}
RESUMO - Nos últimos anos, houve aumento da demanda mundial por fontes alternativas de combustíveis e o sorgo surge como uma planta promissora para a produção de etanol. Portanto, para uma exploração competitiva, a produtividade de biomassa, bem como a sua qualidade, são aspectos que precisam ser considerados. Assim, com este trabalho, objetivou-se avaliar as adubações nitrogenada e potássica sobre a produtividade e a qualidade de fibra de sorgo biomassa para produção de etanol lignocelulósico. Foram instalados experimentos com as cultivares CMSXS 7020 e CMSXS 652 na área experimental da Embrapa Milho e Sorgo em delineamento experimental de blocos ao acaso, com três repetições. Os tratamentos de cada experimento consistiram de um fatorial 4x4, envolvendo quatro doses de $\mathrm{N}\left(0,80,160\right.$ e $\left.240 \mathrm{~kg} \mathrm{ha}^{-1}\right)$ e quatro de $\mathrm{K}_{2} \mathrm{O}\left(0,60,120\right.$ e $\left.180 \mathrm{~kg} \mathrm{ha}^{-1}\right)$ em cobertura. De maneira geral, apenas a matéria seca total do CMSXS 652 foi afetada pelas doses de N, com produtividade máxima na dose de $130 \mathrm{~kg}^{-1}$, não havendo efeito das doses de $\mathrm{K}$. A adubação com $\mathrm{N}$ e $\mathrm{K}$ aumentou os valores de celulose e hemicelulose e reduziu os de lignina e cinzas.
\end{abstract}

Palavras-chave: etanol lignocelulósico; biocombustível; fertilização; nitrogênio; potássio.

\section{NITROGEN NITROGEN AND POTASSIUM FERTILIZATION ON SORGHUM BIOMASS - YIELD AND FIBER QUALITY}

\begin{abstract}
In recent years the worldwide demand for alternative fuel sources has been increasing, and sorghum emerges as a promising plant for the production of ethanol. Therefore, for a competitive exploration, biomass productivity, as well as its fiber quality, some aspects need to be considered. Thus, this study aimed to evaluate the effects of nitrogen and potassium fertilization on yield and fiber quality of sorghum biomass for lignocellulosic ethanol. Experiments were conducted with cultivars CMSXS 7020 and CMSXS 652 in the experimental area of Embrapa Maize and Sorghum in experimental design of randomized blocks with three replications. Treatments of each experiment consisted of a $4 \times 4$ factorial, involving four $\mathrm{N}$ doses $\left(0,80,160\right.$ and $\left.240 \mathrm{~kg} \mathrm{ha}^{-1}\right)$ and four of $\mathrm{K}_{2} \mathrm{O}(0,60,120$ and 180 $\mathrm{kg} \mathrm{ha}^{-1}$ ) topdressed. In general, only the total dry matter of the CMSXS 652 cultivar was affected by $\mathrm{N}$ rates, and maximum yield was obtained with the dose of $130 \mathrm{~kg} \mathrm{ha}^{-1}$, with no effect of doses of $\mathrm{K}$. Fertilization with $\mathrm{N}$ and $\mathrm{K}$ increased the amounts of cellulose and hemicellulose and reduced lignin and ashes.
\end{abstract}

Key words: lignocellulosic ethanol; biofuel; fertilization; nitrogen; potassium. 
A energia de biomassa constitui um importante compartimento do setor para atingir as metas do Programa "Energia Sustentável para Todos", que estipulou 2030 como prazo final para a duplicação do volume atual de $18 \%$ de participação das fontes renováveis na carteira global de energia (United Nations Foundation, 2012).

Contudo, para alcançar o status de fonte limpa, barata e renovável de energia, em oposição àquelas derivadas de petróleo, as culturas envolvidas na produção de biomassa necessitam apresentar um balanço energético positivo, somando-se à eficiência das tecnologias de transformação, ou seja, deve apresentar índice de eficiência energética positivo, que determina quanto de energia é gerada pelo produto para cada unidade de energia fóssil gasta em seu processo de produção (WWF-Brasil, 2009). Nesse sentido, Hill et al. (2009) ressaltam a necessidade de aumentar a eficiência dos sistemas de produção nos cultivos e nas biorrefinarias, já que poucas culturas, como a cana-de-açúcar e o dendê, têm alcançado bons índices de eficiência energética para os sistemas de produção e tecnologias de transformação atuais.

$\mathrm{Na}$ busca de novas alternativas, a cultura do sorgo (Sorghum bicolor (L.) Moench) destacase como uma das matérias-prima mais promissoras para a geração de bioenergia por ser uma cultura amplamente difundida no mundo, de elevado teor de fibras e açúcares fermentáveis, que pode ser explorada em larga escala e com grande adaptabilidade a diversas condições de clima e solo. Além disso, o ciclo curto de produção (seis meses para alto rendimento de biomassa) e a menor exigência em água do que a cana-de-açúcar e o milho favorecem o sorgo, em termos de eficiência energética (Nagaiah et al., 2012; Serna-Saldívar et al., 2012). Mas, para tornar-se viável, ainda é necessário aumentar a produtividade da cultura em relação à quantidade de energia e aos insumos empregados na sua produção. Segundo Urquiaga et al. (2005), enquanto a cana-de-açúcar e o dendê têm apresentado balanços altamente positivos (média de $8,7)$, culturas como o sorgo ainda apresentam baixos retornos, principalmente em relação à adubação nitrogenada, e precisam ser melhoradas mediante seleção de variedades para alto rendimento e substituição ou redução da adubação.

Além disso, as características químicas da biomassa têm influência direta no processo de conversão de energia, sendo que a produção de biocombustíveis lignocelulósicos envolve a despolimerização dos polissacarídeos da parede celular (celulose e hemicelulose) em açúcares simples e fermentáveis, pelos processos de prétratamento e sacarificação, e conversão de açúcares simples em biocombustíveis pela fermentação (Damasceno et al., 2010).

Sendo assim, o Programa de Melhoramento de Sorgo da Embrapa Milho e Sorgo tem desenvolvido linhagens de sorgo visando à elevação do potencial de produção de biomassa. Esses materiais descendem de parentais forrageiros com diferentes características de perfilhamento e desenvolvimento vegetativos que, embora bem estudados quanto a essas características agronômicas, são ainda pouco estudados quanto à eficiência de resposta ao incremento de fertilizantes para altas produtividades e à qualidade de biomassa.

Levando-se em conta que os nutrientes de maior demanda para a produção de biomassa são o nitrogênio $(\mathrm{N})$ e o potássio $(\mathrm{K})$, com este trabalho objetivou-se avaliar as adubações nitrogenada e potássica na produtividade e a qualidade de fibra de 
duas variedades de sorgo biomassa para produção de etanol lignocelulósico.

\section{Material e Métodos}

Foram conduzidos dois experimentos no Campo Experimental da Embrapa Milho e Sorgo, em Sete Lagoas, MG (-19'28' S e $\left.44^{\circ} 15^{\prime} \mathrm{W}\right)$, nas safras 2010-11 e 2011-12, sob irrigação, em Latossolo Vermelho distroférrico típico, com as seguintes características químicas e física: $\mathrm{pH}_{2} \mathrm{O}=5,1, \mathrm{Al}=$ 0,$53 ; \mathrm{Ca}=2,6 ; \mathrm{Mg}=0,6 ; \mathrm{T}=9,8\left(\mathrm{cmol}_{\mathrm{c}} \mathrm{dm}^{-3}\right) ; \mathrm{P}=$ 2,$1 ; \mathrm{K}=39,0\left(\mathrm{mg} \mathrm{dm}^{-3}\right) ; \mathrm{V}=30,0 \%$; teor de argila e matéria orgânica $=67,0$ e 2,7 $\left(\right.$ dag $\left.\mathrm{kg}^{-1}\right)$. O solo recebeu 3,0 t ha-1 de calcário dolomítico (PRNT 95 \%), no dia 22/12/2010, para correção da acidez e elevação da saturação por bases a $60 \%$.

$\mathrm{O}$ delineamento experimental utilizado foi o de blocos ao acaso, com três repetições. Os tratamentos de cada safra consistiram de um fatorial $4 \times 4$, envolvendo quatro doses de $\mathrm{N}(0,80,160$ e 240 $\left.\mathrm{kg} \mathrm{ha}^{-1}\right)$ e quatro de $\mathrm{K}_{2} \mathrm{O}\left(0,60,120\right.$ e $\left.180 \mathrm{~kg} \mathrm{ha}^{-1}\right)$ em cobertura. As parcelas foram constituídas por quatro linhas de $5 \mathrm{~m}$ de comprimento e espaçadas em $0,7 \mathrm{~m}$. A parcela útil foi composta pelas duas linhas centrais de $4 \mathrm{~m}$ de comprimento, eliminandose $0,5 \mathrm{~m}$ de bordadura em cada extremidade.

Utilizaram-se as variedades de sorgo CMSXS 7020 e CMSXS 652, tipo forrageiros e sensíveis ao fotoperíodo, prevendo um estande de 130.000 plantas por hectare (nove plantas por metro linear). Na safra 2010-11, o plantio foi realizado em $02 / 02 / 11$. A adubação de plantio foi de $500 \mathrm{~kg} \mathrm{ha}^{-1}$ do formulado 08-28-16+Zn, aplicados no sulco de semeadura, somados a $17,6 \mathrm{~kg} \mathrm{ha}^{-1}$ de ácido bórico. A adubação de cobertura com NK foi realizada aos 35 dias após o plantio, quando as plantas apresentavam
4 a 6 folhas completamente desenvolvidas. Utilizaram-se as fontes ureia e cloreto de potássio, aplicadas superficialmente e ao lado, à distância de $20 \mathrm{~cm}$, das linhas de plantio. A colheita foi realizada em 10/06/11.

Na safra 2011-12, o plantio foi realizado em 11/11/11 e as adubações (plantio e cobertura) foram as mesmas da safra anterior. A colheita do sorgo CMSXS 7020 foi realizada em 03/05/12 e a do CMSXS 652 em 16/05/12.

As variáveis avaliadas foram produtividade de matéria seca total (MST) (Tabela 1), matéria seca de colmos (COL), folhas (FOL) e panículas (PAN), com suas respectivas porcentagens em relação à produtividade total. $\mathrm{O}$ processo de secagem da matéria verde foi feito em estufa de ventilação forçada a $65{ }^{\circ} \mathrm{C}$ por cerca de cinco dias.

Foi avaliada também a qualidade da fibra, por meio da análise da lignina-LIG, celulose-CEL, hemicelulose-HEM e cinzas totais-CIN (Tabela 2). Utilizaram-se as metodologias de Robertson \& Van Soest (1981) para determinação da lignina, fibra detergente neutro (FDN) e ácido (FDA), sendo que a celulose foi obtida pela diferença da FDA-lignina. A hemicelulose foi obtida pela diferença de FDNFDA. As cinzas totais foram determinadas pelo método de Association (1995).

Os dados foram submetidos à análise de variância (Anova) conjunta em relação às duas safras para testar os efeitos de $\mathrm{N}$ e K, safra e as respectivas interações. Foram ajustadas regressões para os efeitos significativos ( $p<0,05)$, utilizandose o programa estatístico SAS.

Para a variável MST, quando houve ajuste de equações quadráticas, foram calculadas a dose de máxima eficiência física (igualando a primeira derivada da equação quadrática a zero) e a dose 
TABELA 1. Valores médios de produtividade de matéria seca total (MST) e dos seus componentes: colmo (COL), folha (FOL) e panícula (PAN) em porcentagem, ao final da colheita, nas safras 2010-2011 e 2011-2012 (1 e 2, respectivamente), como variáveis das doses de NK. CMSXS 7020.

\begin{tabular}{|c|c|c|c|c|c|c|c|c|c|}
\hline \multirow{2}{*}{$\mathbf{N}$} & \multirow{2}{*}{$\mathrm{K}_{2} \mathrm{O}$} & \multirow{2}{*}{ MST1 } & \multirow{2}{*}{ MST2 } & \multicolumn{6}{|c|}{ Matéria Seca } \\
\hline & & & & COL1 & FOL1 & PAN1 & COL2 & FOL2 & PAN2 \\
\hline \multicolumn{2}{|c|}{----- $\mathrm{kg} \mathrm{ha}^{-1}$----- } & \multicolumn{2}{|c|}{ 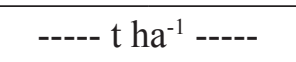 } & \multicolumn{6}{|c|}{ 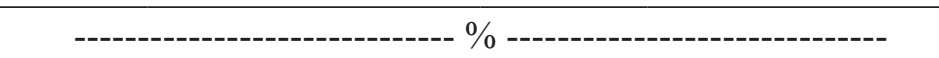 } \\
\hline 0 & 0 & 11,32 & 16,21 & 77,67 & 19,14 & 3,19 & 72,71 & 21,02 & 6,27 \\
\hline 0 & 60 & 11,05 & 13,75 & 77,13 & 17,89 & 4,99 & 75,06 & 19,03 & 5,91 \\
\hline 0 & 120 & 10,38 & 17,86 & 80,17 & 17,46 & 2,36 & 74,22 & 19,56 & 6,21 \\
\hline 0 & 180 & 12,93 & 20,35 & 79,53 & 17,56 & 2,91 & 75,51 & 19,29 & 5,20 \\
\hline 80 & 0 & 11,07 & 16,34 & 79,42 & 17,28 & 3,30 & 75,73 & 20,30 & 3,97 \\
\hline 80 & 60 & 11,75 & 21,56 & 79,87 & 16,99 & 3,13 & 75,46 & 18,68 & 5,86 \\
\hline 80 & 120 & 12,56 & 18,46 & 76,86 & 17,94 & 5,20 & 75,10 & 18,95 & 5,94 \\
\hline 80 & 180 & 15,45 & 20,09 & 77,57 & 18,08 & 4,35 & 78,07 & 17,39 & 4,54 \\
\hline 160 & 0 & 12,89 & 20,16 & 78,57 & 17,84 & 3,59 & 75,47 & 18,35 & 6,18 \\
\hline 160 & 60 & 13,58 & 18,82 & 78,23 & 19,25 & 2,52 & 76,40 & 17,12 & 6,49 \\
\hline 160 & 120 & 12,89 & 18,10 & 78,08 & 18,73 & 3,19 & 76,83 & 18,43 & 4,74 \\
\hline 160 & 180 & 14,19 & 19,22 & 79,90 & 17,48 & 2,61 & 74,92 & 19,41 & 5,67 \\
\hline 240 & 0 & 11,71 & 15,07 & 78,31 & 19,25 & 2,44 & 76,76 & 17,98 & 5,27 \\
\hline 240 & 60 & 13,50 & 19,59 & 80,36 & 17,05 & 2,59 & 74,92 & 18,50 & 6,58 \\
\hline 240 & 120 & 13,10 & 22,24 & 77,63 & 17,11 & 5,25 & 75,13 & 18,05 & 6,83 \\
\hline 240 & 180 & 10,90 & 15,92 & 79,39 & 17,55 & 3,06 & 76,34 & 17,69 & 5,97 \\
\hline
\end{tabular}

de máxima eficiência econômica (dose estimada considerando $90 \%$ da produtividade máxima de MST de sorgo).

\section{Resultados e Discussão}

O baixo estande da safra 2010-2011 (média de sete plantas por metro linear), somado ao plantio tardio (fevereiro de 2011), refletiram numa baixa produtividade de matéria seca do CMSXS 7020 e do CMSXS 652 (Tabelas 1 e 3, respectivamente). A época de plantio mais indicada para a cultura é de outubro até meados de dezembro, quando o sorgo pode expressar melhor o seu potencial produtivo. Dados de Parrella et al. (2010), em pesquisa com os mesmos materiais plantados em novembro (Sete Lagoas, MG) e dezembro (Nova Porteirinha, MG), no ano de 2009, resultaram em produtividades médias de matéria seca de 22,71 e 33,87 t ha-1 para o CMSXS 7020 e o CMSXS 652, respectivamente, considerando os dois locais. Essa produtividade de matéria seca supera as produtividades relatadas para cultivares de sorgo forrageiro comerciais existentes no mercado, que estão em torno de 15 a $20 \mathrm{t} \mathrm{ha}^{-1}$ (Rodrigues et al., 2008; Skonieski et al., 2010) e também os resultados médios da safra 2010-2011 de matéria seca para o CMSXS 7020 e o CMSXS 652 $\left(12,46\right.$ e $12,50 \mathrm{t} \mathrm{ha}^{-1}$, respectivamente) e safra 2011 2012 (18,36 e 23,13 $\mathrm{tha}^{-1}$, respectivamente) obtidas neste trabalho. 
TABELA 2. Valores médios de lignina (LIG), celulose (CEL), hemicelulose (HEM) e cinzas (CIN), nas safras 2010-2011 e 2011-2012 (1 e 2, respectivamente), como variáveis das doses de NK. CMSXS 7020.

\begin{tabular}{cccccccccc}
\hline $\mathrm{N}$ & $\mathrm{K}_{2} \mathrm{O}$ & LIG1 & CEL1 & HEM1 & CIN1 & LIG2 & CEL2 & HEM2 & CIN2 \\
\hline$----k g ~ h h^{-1}----$ & $----------------------------------\%$----------------------------------- \\
0 & 0 & 6,30 & 38,30 & 25,65 & 3,71 & 5,49 & 35,67 & 30,30 & 3,58 \\
0 & 60 & 6,04 & 36,83 & 26,36 & 3,93 & 6,50 & 37,51 & 29,16 & 3,07 \\
0 & 120 & 5,61 & 38,32 & 24,87 & 3,40 & 6,28 & 36,85 & 29,82 & 3,49 \\
0 & 180 & 7,08 & 38,45 & 25,92 & 3,72 & 6,59 & 38,48 & 30,41 & 3,79 \\
80 & 0 & 5,63 & 36,94 & 26,36 & 4,01 & 5,62 & 36,68 & 31,12 & 3,42 \\
80 & 60 & 6,74 & 38,48 & 24,92 & 4,04 & 7,44 & 39,45 & 28,76 & 3,62 \\
80 & 120 & 6,70 & 39,00 & 24,10 & 4,16 & 6,35 & 38,15 & 29,73 & 3,75 \\
80 & 180 & 5,56 & 36,82 & 26,22 & 3,95 & 7,10 & 39,26 & 29,48 & 3,37 \\
160 & 0 & 6,91 & 38,42 & 24,81 & 3,63 & 6,02 & 38,17 & 30,55 & 3,13 \\
160 & 60 & 5,57 & 36,37 & 27,62 & 3,44 & 6,63 & 38,02 & 29,70 & 3,21 \\
160 & 120 & 5,42 & 36,69 & 26,34 & 3,82 & 6,73 & 38,16 & 29,69 & 3,50 \\
160 & 180 & 6,65 & 36,47 & 25,96 & 3,48 & 7,04 & 38,85 & 29,29 & 3,09 \\
240 & 0 & 6,80 & 37,99 & 24,15 & 3,36 & 7,50 & 37,45 & 28,83 & 2,84 \\
240 & 60 & 5,50 & 37,56 & 25,57 & 3,67 & 6,51 & 37,48 & 30,25 & 3,19 \\
240 & 120 & 6,87 & 40,65 & 22,57 & 3,91 & 6,93 & 37,16 & 29,22 & 3,12 \\
240 & 180 & 6,50 & 37,52 & 25,02 & 3,76 & 6,34 & 38,67 & 30,42 & 3,42 \\
\hline
\end{tabular}

Contudo, os resultados da produtividade de matéria seca total dos materiais na safra 20112012 se aproximam de outros trabalhos com investigação do potencial de sorgo para produção de biocombustíveis, como no de Hoffmann Jr. \& Rooney (2012), que obtiveram uma média de 23,53 $\mathrm{t} \mathrm{ha}^{-1}$ de matéria seca envolvendo seis genótipos de sorgo lignocelulósico; e no de Amaducci et al. (2000), em que o sorgo produziu 26,2 $\mathrm{t} \mathrm{ha}^{-1}$ de matéria seca, superando as produtividades do milho, kenaf e cânhamo.

O ciclo do CMSXS 7020 e do CMSXS 652 obtido nesta pesquisa na safra 2010-2011 foi de cerca de 128 dias após o plantio, com ocorrência do florescimento aos 76 dias. Já na safra 2011-2012, o ciclo dos materiais foi de cerca de 180 dias após o plantio, com florescimento por volta de 155 dias.
No trabalho de Parrella et al. (2010) e Parrella et al. (2011), os materiais floresceram com 147 dias (média dos dois locais) e 138 dias (média de três locais), respectivamente, ou seja, passaram bem mais tempo no campo acumulando biomassa, à semelhança do que ocorreu com os materiais deste trabalho na safra 2011-2012. Essa variação de ciclo dos materiais sensíveis ao fotoperíodo é um fator positivo a ser explorado no cultivo do sorgo para potencializar a produção de biomassa e que, aliado a outras características, evidencia o quanto promissor é o sorgo para a produção de biocombustíveis.

A análise de variância para o CMSXS 7020 mostrou que houve efeito significativo apenas das doses de $\mathrm{N}$ para CIN, efeito da interação das doses de N x K para a MST e para a LIG e efeito de safra $x \mathrm{~N}$ e safra $\mathrm{x} K$ para CEL, sendo apresentadas as 
equações de regressão com ajustes significativos $(\mathrm{p}<0,05)$ na Tabela 5. Para o CMSXS 652, houve efeito das doses de $\mathrm{N}$ sobre a MST, das doses de $\mathrm{K}$ sobre a CEL e CIN e da interação das doses de NxK para a HEM, com as equações de regressão mostradas na Tabela 5.

Embora a Anova tenha resultado em efeito não significativo $(\mathrm{p}<0,05)$ para as interações das doses de K/N0 e K/N80 para o CMSXS 7020, houve ajuste significativo de equações lineares $\left(\mathrm{K} / \mathrm{N} 0-\mathrm{Y}=12,68+0,017236 * \mathrm{X}, \mathrm{R}^{2}=57,03\right.$ e K/ $\left.\mathrm{N} 80-\mathrm{Y}=14,25+0,018425^{*} \mathrm{X}, \mathrm{R}^{2}=67,46\right)$.

Pela equação da Figura 1A (N/K60), é possível determinar que a máxima produtividade de MST para o CMSXS 7020 foi obtida com a dose de 168,72 $\mathrm{kg} \mathrm{ha}^{-1}$ de $\mathrm{N}$. Da mesma forma, a dose de máxima eficiência econômica $(90 \%$ da máxima eficiência física) seria de $63,23 \mathrm{~kg} \mathrm{ha}^{-1} \mathrm{de}$ N. Para N/K180 (Figura 1B), a dose de máxima eficiência física $\left(17,77 \mathrm{t} \mathrm{ha}^{-1}\right)$ seria de $81,09 \mathrm{~kg}$ ha $^{-1}$ de $\mathrm{N}$ e a máxima eficiência econômica já seria atingida sem aplicação de N. Já a dose de K/N240 (Figura 1C) para a máxima produtividade de sorgo seria de $91,86 \mathrm{~kg} \mathrm{ha}^{-1}$ e para a máxima eficiência econômica de 33,46 $\mathrm{kg} \mathrm{ha}^{-1}$.

Para o CMSXS 652, a máxima produtividade de MST de sorgo (19,21 t ha-1) foi obtida com a dose de $148,3 \mathrm{~kg} \mathrm{ha}^{-1}$ de $\mathrm{N}$ e a dose de máxima eficiência econômica foi de $39,06 \mathrm{~kg}$ ha $^{-1}$ de N (Figura 1D).

TABELA 3. Valores médios de produtividade de matéria seca total (MST) e dos seus componentes: colmo (COL), folha (FOL) e panícula (PAN) em porcentagem, ao final da colheita, nas safras 2010-2011 e 2011-2012 (1 e 2, respectivamente), como variáveis das doses de NK. CMSXS 652.

\begin{tabular}{|c|c|c|c|c|c|c|c|c|c|}
\hline \multirow{2}{*}{$\mathbf{N}$} & \multirow{2}{*}{$\mathbf{K}_{2} \mathbf{O}$} & \multirow{2}{*}{ MST1 } & \multirow{2}{*}{ MST2 } & \multicolumn{6}{|c|}{ Matéria Seca } \\
\hline & & & & COL1 & FOL1 & PAN1 & COL2 & FOL2 & PAN2 \\
\hline \multicolumn{2}{|c|}{----- $\mathrm{kg} \mathrm{ha}^{-1}$----- } & \multicolumn{2}{|c|}{----- $\mathrm{t} \mathrm{ha}^{-1}-----$} & \multicolumn{6}{|c|}{ 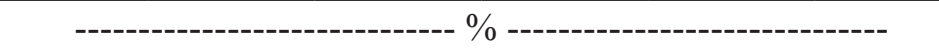 } \\
\hline 0 & 0 & 10,73 & 20,03 & 71,64 & 23,87 & 4,49 & 69,43 & 20,04 & 10,53 \\
\hline 0 & 60 & 11,23 & 19,04 & 79,99 & 17,22 & 2,79 & 69,83 & 20,84 & 9,33 \\
\hline 0 & 120 & 9,40 & 19,87 & 74,49 & 21,64 & 3,87 & 67,89 & 20,83 & 11,29 \\
\hline 0 & 180 & 13,15 & 19,08 & 75,33 & 18,79 & 5,88 & 68,96 & 20,78 & 10,26 \\
\hline 80 & 0 & 11,79 & 25,50 & 77,27 & 19,81 & 2,92 & 66,90 & 22,29 & 10,81 \\
\hline 80 & 60 & 12,14 & 23,36 & 78,74 & 18,27 & 2,99 & 68,10 & 19,67 & 12,22 \\
\hline 80 & 120 & 16,38 & 21,56 & 75,05 & 19,45 & 5,50 & 69,59 & 19,09 & 11,32 \\
\hline 80 & 180 & 11,77 & 33,95 & 73,38 & 22,05 & 4,57 & 71,03 & 19,71 & 9,26 \\
\hline 160 & 0 & 13,85 & 22,63 & 77,24 & 19,23 & 3,53 & 70,52 & 20,81 & 8,67 \\
\hline 160 & 60 & 14,60 & 23,36 & 75,80 & 21,56 & 2,64 & 71,41 & 20,96 & 7,63 \\
\hline 160 & 120 & 12,35 & 20,23 & 76,78 & 18,68 & 4,54 & 68,99 & 19,73 & 11,28 \\
\hline 160 & 180 & 15,42 & 22,53 & 78,97 & 17,90 & 3,13 & 67,66 & 21,27 & 11,06 \\
\hline 240 & 0 & 12,64 & 23,11 & 76,66 & 20,31 & 3,02 & 71,56 & 19,36 & 9,08 \\
\hline 240 & 60 & 12,36 & 19,41 & 76,43 & 19,16 & 4,41 & 67,77 & 20,85 & 11,38 \\
\hline 240 & 120 & 10,71 & 26,71 & 75,95 & 20,69 & 3,37 & 70,07 & 20,05 & 9,89 \\
\hline 240 & 180 & 11,34 & 29,71 & 78,53 & 17,34 & 4,12 & 69,86 & 21,02 & 9,13 \\
\hline
\end{tabular}



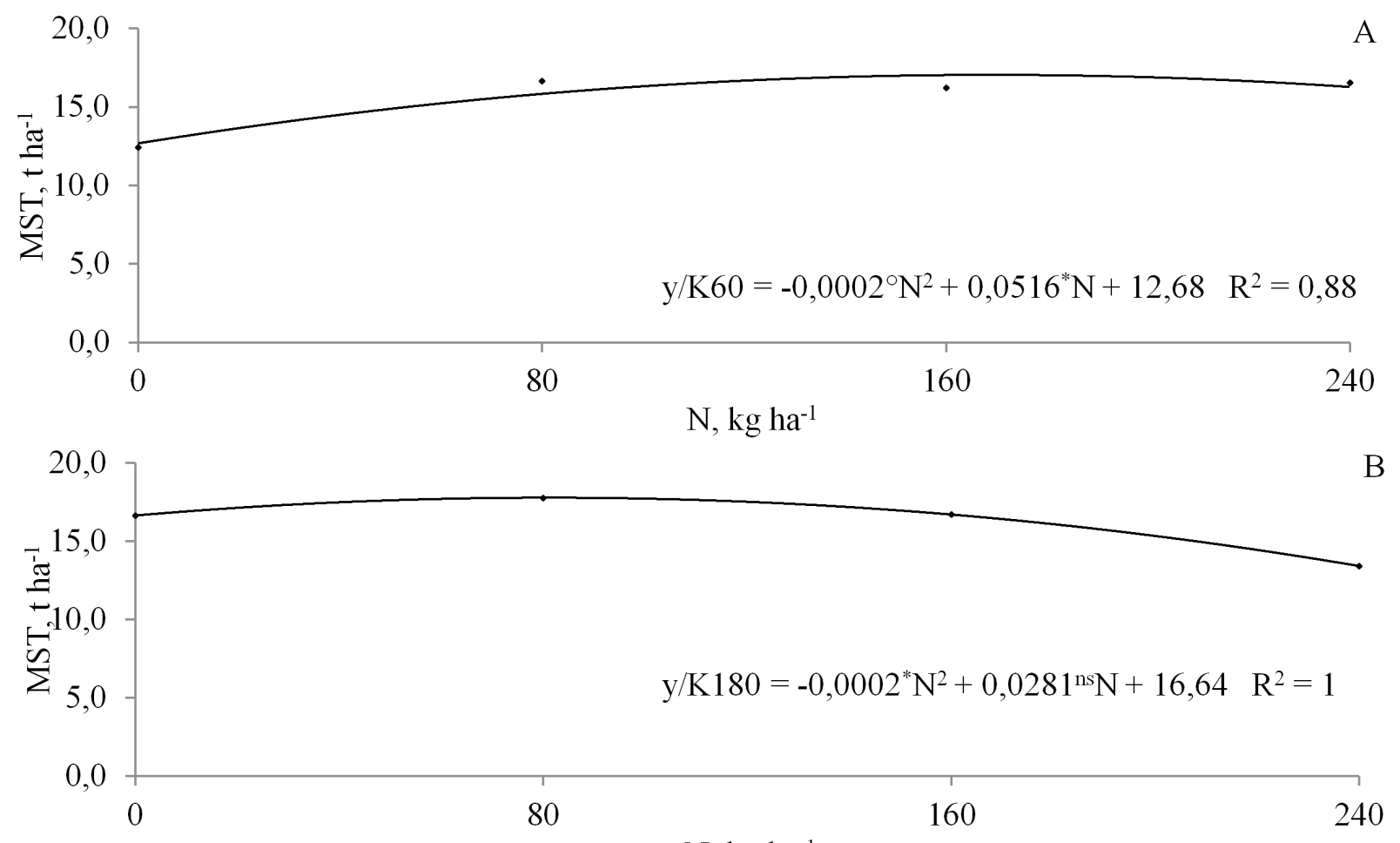

$\mathrm{N}, \mathrm{kg} \mathrm{ha}^{-1}$
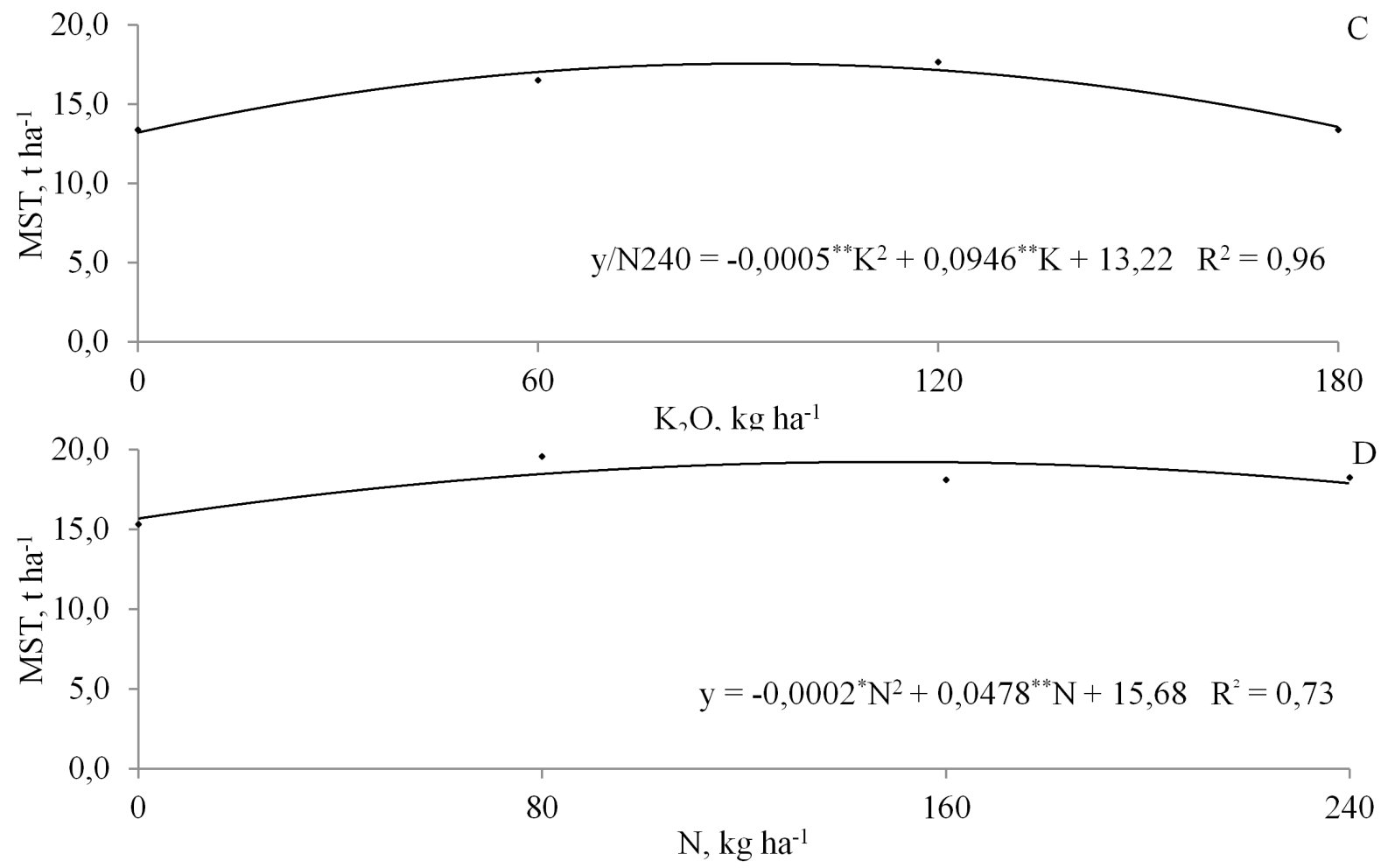

FIGURA 1. Gráficos dos modelos de regressão ajustados para a produtividade de matéria seca total (MST) como variável das doses de $\mathrm{N}$ (A e B) e $\mathrm{K}_{2} \mathrm{O}$ (C) para o CMSXS 7020 e das doses de N (D) para o CMSXS 652. 
Os valores razoavelmente baixos de doses econômicas de $\mathrm{N}$ e $\mathrm{K}$, nas condições destes experimentos, evidenciam que o custo com adubações nitrogenada e potássica poderia ser bem reduzido, sem afetar significativamente a produtividade de matéria seca do sorgo. A contribuição de formas nãotrocáveis de K do solo (Melo et al., 2005) e a liberação de $\mathrm{N}$ da matéria orgânica do solo (cada 1 dag $\mathrm{kg}^{-1}$ de matéria orgânica pode fornecer cerca de $30 \mathrm{~kg} \mathrm{ha}^{-1}$ de N (Sousa \& Lobato, 2004) - solo com 2,7 dag $\mathrm{kg}^{-1}$ de matéria orgânica $=81 \mathrm{~kg} \mathrm{ha}^{-1}$ de $\mathrm{N}$ ), aliadas à esperada liberação de $\mathrm{N}$ e $\mathrm{K}$ do resíduo vegetal da área (dados não medidos), podem justificar, em parte, estas constatações. Adicionalmente, o sorgo é reconhecido pela alta eficiência na utilização do N. Gardner et al. (1994), Maranville \& Madhavan (2002) e Barbanti et al. (2006) relatam que o sorgo pode produzir cerca de $20 \mathrm{t} \mathrm{ha}^{-1}$ de matéria seca apenas com a reserva de $\mathrm{N}$ do solo. Os resultados obtidos neste trabalho em parte corroboram os de Rodrigues Filho et al. (2006), em estudo com quatro híbridos de sorgo forrageiro, que não encontraram diferença significativa $(\mathrm{p}<0,05)$ na produção de matéria seca em função das doses de $\mathrm{N}$ entre $50 \mathrm{e}$ $100 \mathrm{~kg} \mathrm{ha}^{-1}$, cujas médias foram de 59,32 e 15,17 $\mathrm{t} \mathrm{ha}^{-1}$, respectivamente. Entretanto, há relatos de produtividade de matéria seca bem mais elevada em plantios de sorgo biomassa (cerca de 60 a $90 \mathrm{t} \mathrm{ha}^{-1}$, comunicação pessoal), quando plantados em épocas mais adequadas (setembro, outubro), de forma que pode-se inferir que a predominância de falta de resposta dos materiais estudados às doses de NK pode ser justificada, também, pelo plantio fora da época mais indicada para a cultura, quando o sorgo poderia expressar melhor o seu potencial produtivo. Ou seja, se plantados mais cedo, os materiais acumulariam mais biomassa e, consequentemente, aumentariam a demanda em nutrientes e seria esperada resposta mais pronunciada às doses de $\mathrm{N}$ e $\mathrm{K}$.

A análise da porcentagem de cada componente da matéria seca (colmo, folha e panícula) é importante para o cultivo de sorgo biomassa, em que objetiva-se maior produção de colmo. Neste trabalho, observou-se que a porcentagem de colmo variou de 73 a $80 \%$ da matéria seca (Tabelas 1 e 3), embora essas porcentagens não tenham sofrido efeito da adubação N x K. A baixa porcentagem de panícula em relação à matéria seca total da planta pode ser justificada pelo ataque de pássaros aos grãos da panícula, de forma que as parcelas produziram muito pouco (Tabelas 1 e 3 ).

Em relação às variáveis de qualidade de fibra, é importante ressaltar que a celulose e a hemicelulose são os principais componentes da biomassa e, sob hidrólise enzimática, produzem açúcares fermentáveis para a produção de biocombustíveis (Nagaiah et al., 2012). Dessa forma, valores maiores desses componentes são desejáveis na biomassa do sorgo. Por outro lado, esperam-se valores menores de lignina e cinzas para se obter um melhor rendimento em biocombustível.

A lignina é um componente estrutural da parede celular que fornece força às plantas e também proteção ao ataque de pragas. A lignina pode afetar o processo de sacarificação da biomassa em açúcares fermentáveis de duas maneiras: ela pode adsorver irreversivelmente as enzimas hidrolíticas, bloqueando sua ação na celulose (Converse, 1993; Palonen et al., 2004), e, por ser hidrofóbica, pode também prevenir que a fibra celulósica se entumeça, o que diminui a área de superfície acessível às enzimas (Mooney et al., 1998). Com isso, há necessidade de maiores concentrações das enzimas para o processamento da biomassa, tornando-o 
economicamente inviável. As cinzas decrescem a eficiência da combustão. Isso ocorre devido ao fato de elas diminuírem a transferência de calor (Burner et al., 2009).

De maneira geral, quando houve efeito da adubação NK sobre as variáveis de qualidade de fibra, evidencia-se efeito positivo no sentido de aumentar as variáveis benéficas (Tabelas 2 e 5) e diminuir as prejudiciais (Tabelas 4 e 5). Isso mostra a importância da adubação, não apenas no aumento de produtividade, mas também na melhoria da qualidade do material, o que favorece o processo de conversão de matéria seca em biocombustíveis.

Neste trabalho, a porcentagem de lignina do CMSXS 7020, considerando as duas safras, variou de 5,4 a 7,5, com média de 6,4, e o CMSXS 652 teve a porcentagem de lignina variando de 5,1 a
7,3 e valor médio de 6,2. Trabalho de Amaducci et al. (2000) encontrou valor médio de lignina para o sorgo em torno de $8 \%$, ou seja, acima dos valores encontrados para os materiais em estudo nesta pesquisa. Os mesmos autores obtiveram valores de 39 a $47 \%$ de celulose e de 26 a $29 \%$ de hemicelulose para o sorgo, enquanto neste trabalho os valores de celulose variaram de 35,6 a $40,6 \%$ para o CMSXS 7020 e de 33,3 a $41,2 \%$ para o CMSXS 652, considerando as duas safras. Enquanto na pesquisa de Nagaiah et al. (2012), os valores de lignina se estenderam de 6,2 a $8,1 \%$, os de celulose de 27 a $52 \%$ e os de hemicelulose de 17 a $23 \%$. A comparação da amplitude de valores para esses três principais componentes da matéria seca do sorgo mostra que não há uma variação muito grande e o trabalho de Serna-Saldívar et al. (2012) resultou em valores

TABELA 4. Valores médios de lignina (LIG), celulose (CEL), hemicelulose (HEM) e cinzas (CIN), nas safras 2010-2011 e 2011-2012 (1 e 2, respectivamente), como variáveis das doses de NK. CMSXS 652.

\begin{tabular}{|c|c|c|c|c|c|c|c|c|c|}
\hline $\mathrm{N}$ & $\mathrm{K}_{2} \mathrm{O}$ & LIG1 & CEL1 & HEM1 & CIN1 & LIG2 & CEL2 & HEM2 & CIN2 \\
\hline \multicolumn{2}{|c|}{----kg ha"-1---- } & \multicolumn{8}{|c|}{------------------------------------------------0\%"------------------------------------------------- } \\
\hline 0 & 0 & 6,14 & 39,81 & 25,54 & 3,74 & 6,30 & 35,27 & 30,61 & 3,24 \\
\hline 0 & 60 & 5,26 & 39,52 & 26,59 & 3,97 & 6,84 & 33,55 & 29,33 & 3,30 \\
\hline 0 & 120 & 6,36 & 39,82 & 27,53 & 4,26 & 6,47 & 35,33 & 30,66 & 3,16 \\
\hline 0 & 180 & 5,71 & 41,22 & 26,83 & 4,35 & 6,16 & 35,67 & 30,78 & 3,38 \\
\hline 80 & 0 & 6,00 & 40,52 & 25,97 & 3,92 & 6,75 & 36,29 & 28,65 & 2,63 \\
\hline 80 & 60 & 5,25 & 38,52 & 28,09 & 4,22 & 7,37 & 35,15 & 30,34 & 3,40 \\
\hline 80 & 120 & 6,00 & 41,00 & 25,19 & 5,10 & 6,79 & 33,94 & 29,16 & 3,38 \\
\hline 80 & 180 & 5,61 & 40,12 & 27,14 & 4,07 & 7,27 & 35,97 & 29,39 & 2,95 \\
\hline 160 & 0 & 6,14 & 40,15 & 27,83 & 3,67 & 6,32 & 33,33 & 30,63 & 2,72 \\
\hline 160 & 60 & 5,84 & 38,87 & 25,42 & 4,07 & 6,91 & 34,49 & 29,52 & 3,04 \\
\hline 160 & 120 & 5,78 & 40,24 & 28,15 & 4,04 & 6,87 & 35,99 & 30,73 & 3,18 \\
\hline 160 & 180 & 5,34 & 39,57 & 28,00 & 4,15 & 6,76 & 35,88 & 30,46 & 2,93 \\
\hline 240 & 0 & 5,64 & 39,31 & 27,85 & 4,11 & 6,98 & 34,24 & 29,79 & 2,86 \\
\hline 240 & 60 & 5,58 & 40,22 & 28,50 & 4,86 & 6,57 & 33,83 & 31,00 & 2,81 \\
\hline 240 & 120 & 5,88 & 39,96 & 27,16 & 4,54 & 6,85 & 36,03 & 30,30 & 3,24 \\
\hline 240 & 180 & 5,10 & 39,19 & 26,77 & 4,23 & 6,61 & 34,54 & 30,56 & 2,94 \\
\hline
\end{tabular}


TABELA 5. Modelos de regressão ajustados para as diferentes variáveis com efeito significativo na análise de variância para o CMSXS 7020 e o CMSXS 652.

\begin{tabular}{|c|c|c|c|}
\hline Variável & Fonte de variação & Equação & $\mathbf{R}^{2}$ \\
\hline \multicolumn{4}{|c|}{ CMSXS 7020} \\
\hline \multirow{2}{*}{ LIG } & $\mathrm{N} / \mathrm{K} 0$ & $\mathrm{y}=0,0057^{* *} \mathrm{~N}+5,59$ & 0,77 \\
\hline & $\mathrm{K} / \mathrm{N} 80$ & $\mathrm{y}=-0,0001^{*} \mathrm{~K}^{2}+0,0233^{*} \mathrm{~K}+5,74$ & 0,74 \\
\hline \multirow{4}{*}{ CEL } & $\mathrm{N} /$ safra 1 & sem ajuste & \\
\hline & $\mathrm{N} /$ safra 2 & $\mathrm{y}=-0,00007^{*} \mathrm{~N}^{2}+0,019462^{*} \mathrm{~N}+37,16$ & 0,97 \\
\hline & $\mathrm{K} /$ safra 1 & sem ajuste & \\
\hline & $\mathrm{K} /$ safra 2 & $\mathrm{y}=0,0082 * \mathrm{~K}+37,13$ & 0,67 \\
\hline CIN & $\mathrm{N}$ & $\mathrm{y}=-0,0011^{*} \mathrm{~N}+3,68$ & 0,43 \\
\hline \multicolumn{4}{|c|}{ CMSXS 652} \\
\hline \multirow{4}{*}{ HEM } & $\mathrm{N} / \mathrm{K} 60$ & sem ajuste & \\
\hline & $\mathrm{N} / \mathrm{K} 120$ & sem ajuste & \\
\hline & $\mathrm{K} / \mathrm{N} 80$ & sem ajuste & \\
\hline & $\mathrm{K} / \mathrm{N} 160$ & sem ajuste & \\
\hline CEL & $\mathrm{K}$ & $\mathrm{y}=0,0037^{\circ} \mathrm{K}+37,08$ & 0,36 \\
\hline $\mathrm{CIN}$ & $\mathrm{K}$ & $\mathrm{y}=-0,00004^{* *} \mathrm{~K} 2+0,0088^{* * *} \mathrm{~K}+3,35$ & 0,99 \\
\hline
\end{tabular}

bem mais baixos de lignina $(7 \%)$ e cinzas $(0,7 \%)$ do sorgo biomassa em comparação com a cana, o milho, o trigo e o arroz. Por outro lado, o sorgo biomassa foi um pouco inferior quando avaliadas a celulose e a hemicelulose; nesta última, superando apenas a cana-de-açúcar. Os resultados, em parte, reforçam o potencial do sorgo para produção de biocombustíveis, uma vez que sua biomassa apresenta características favoráveis à conversão energética.

\section{Conclusões}

Pelos resultados obtidos neste trabalho, a dose de $\mathrm{N}$ para a obtenção da máxima eficiência econômica, considerando o sorgo CMSXS 7020, foi cerca de $60 \mathrm{~kg} \mathrm{ha}^{-1}$, enquanto para o CMSXS 652 a dose econômica de $\mathrm{N}$ foi de, aproximadamente, $40 \mathrm{~kg} \mathrm{ha}^{-1}$. Para o K, a dose de máxima eficiência econômica foi de $30 \mathrm{~kg} \mathrm{ha}^{-1}$ para o CMSXS 7020. A adubação NK, em geral, teve efeito positivo sobre as variáveis de qualidade de fibra.

\section{Agradecimentos}

À Fundação de Amparo à Pesquisa do Estado de Minas Gerais (Fapemig), pelo apoio financeiro.

\section{References}

ALLIANZ BRASIL. O impacto do mercado expansão da agricultura brasileira e suas consequências para as mudanças climáticas. Disponível em: <http://assets. ww fbr.panda.org/downloads/relatorio biocombustiveis_wwf_brasil_jul09.pdf $>$. Acesso em: 18 maio 2010. 
AMADUCCI, S.; AMADUCCI, R.; BENATI, R.; VENTURI, G. Crop yield and quality parameters of four annual fibre crops (hemp, kenaf, maize and sorghum) in the North of Italy. Industrial Crops and Products, Philadelphia, v. 11, p. 179-186, 2000.

ASSOCIATION OF OFFICIAL ANALYTICAL CHEMISTS. Official methods of analysis of the Association of Official Analytical Chemists. 16. ed. Washington, 1995. 2000 p. BARBANTI, L.; GRANDI, S.; VECCHI, A.; VENTURI, G. Sweet and fibre sorghum (Sorghum bicolor (L.) Moench), energy crops in the frame of environmental protection from excessive nitrogen loads. European Journal of Agronomy, Amsterdam, v. 25, p. 30-39, 2006.

BURNER, D.W.; TEW, T.L.; HARVEY, J.J.; BELESKY, D.P. Dry matter partitioning and quality of Miscathus, Panicum, Saccharum genotypes in Arkansas, USA. Biomass Bioenergy, Philadelphia, v. 33, p. 610-619, 2009.

CONVERSE, A. O. Substrate factors limiting enzymatic hydrolysis. In: SADDLER, J. N. (Ed.). Bioconversion of forest and agricultural plant residues. Wallingford: CAB International, 1993. p. 93-105.

DAMASCENO, C. M. B.; SOUSA, S. M.; NODA, R. W.; PARRELLA, R. A. C.; SCHAFFERT, R. E.; MAGALHAES, J. V. de. Aimportância da lignina para a produção de etanol de segunda geração. Sete Lagoas: Embrapa Milho e Sorgo, 2010. 35 p. (Embrapa Milho e Sorgo. Documentos, 108).

GARDNER, J. C.; MARANVILLE, J. W.; PAPAROZZI, E. T. Nitrogen use efficiency among diverse sorghum cultivars. Crop Science, Madison., v. 34, p. 728-733, 1994.

HILL, J.; POLASKY, S.; NELSON, E.; TILMAN, D.; HUO, H.; LUDWIG, L.; NEUMANN, J.; ZHENG, H.; BONTA, D. Climate change and health costs of air emissions from biofuels and gasoline. Proceedings of the National Academy of Sciences of the United States of America, Washington, v. 106, p. 20772082, 2009.

HOFFMANN JR., L.; ROONEY, W. L. Accumulation of biomass and compositional change over the growth season for six photoperiod sorghum lines. In: SUN GRANT NATIONAL CONFERENCE, New Orleans, 2012. Disponível em: <http://sungrant. tennessee.edu/NR/rdonlyres/D0A5701FE268-43 A 9 - B 312 D A C B D B A 937 B 0 /3683/311Hoffmann_Leo.pdf.>. Acesso em: 14 mai 2013.

MARANVILLE, J. W.; MADHAVAN, S. Physiological adaptations for nitrogen use efficiency in sorghum. Plant and Soil, The Hague, v. 245, p. 25-34, 2002.

MELO, V. F.; CORRÊA, G. F.; RIBEIRO, A. N.; MASCHIO, P. A. Cinética de liberação de potássio e magnésio pelos minerais da fração areia de solos do Triângulo Mineiro. Revista Brasileira de Ciencia do Solo, Campinas, v. 29, p. 533-545, 2005.

MOONEY, C. A.; MANSFIELD, S. D.; TOUHY, M. G.; SADDLER, J. N. The effect of initial pore volume and lignin content on the enzymatic hydrolysis of softwoods. Bioresource Technology, Essex, v. 64, p. 113-119, 1998.

NAGAIAH, D.; SRINIVASA RAO, P.; 
PRAKASHAM, R. S.; UMA, A.; RADHIKA, K.; BARVE, Y.; UMAKANTH, A. V. High biomass sorghum as a potential raw material for biohydrogen production: a preliminar avaluation. Current Trends in Biotechnology and Pharmacy, Guntur, v. 6. p. 183-189, 2012.

PALONEN, H.; TJERNELD, F.; ZACCHI, G.; TENKANEN, M. Adsorption of Trichoderma reesei $\mathrm{CBH}$ I and EG II and their catalytic domains on steam pretreated softwood and isolated lignin. Journal of Biotechnology, Amsterdam, v. 107, p. 65-72, 2004.

PARRELLA, R. A. C.; SCHAFFERT, R. E.; MAY, A.; EMYGDIO, B.; PORTUGAL, A. F.; DAMASCENO, C. M. B. Desempenho agronômico de híbridos de sorgo biomassa. Sete Lagoas: Embrapa Milho e Sorgo, 2011. 19 p. (Embrapa Milho e Sorgo. Boletim de Pesquisa e Desenvolvimento, 41).

PARRELlA, R. A.C.; RODRIGUES, J. A. S.; TARDIN, F. D.; DAMASCENO, C. M. B.; SCHAFFERT, R. E. Desenvolvimento de híbridos de sorgo sensíveis ao fotoperíodo visando alta produtividade de biomassa. Sete Lagoas: Embrapa Milho e Sorgo, 2010. 25 p. (Embrapa Milho e Sorgo. Boletim de Pesquisa e Desenvolvimento, 28).

ROBERTSON, J. B.; VAN SOEST, P. J. The detergent system of analysis and its application to humans foods. In: JAMES, H. P. T.; THEANDER, O. (Ed.). The analysis of dietary fiber in food. New York, Marcel Dekker, 1981. p. 123-158.

RODRIGUES FILHO, O.; FRANÇA, A. F. S.; OLIVEIRA， R. P.; OLIVEIRA， E. R.; ROSA, B.; SOARES, T. V.; MELLO, S. Q.
S. Produção e composição bromatológica de quatro híbridos de sorgo forrageiro [Sorghum bicolor (L.) Moench] submetidos a três doses de nitrogênio. Ciência Animal Brasileira, Goiânia, v. 7, p. 37-48, 2006.

RODRIGUES, J. A. S.; SANTOS, F. G.; SHAFFERT, R. E.; FERREIRA, A. da S.; CASELA, C. R.; TARDIN, F. D. BRS 655: híbrido de sorgo forrageiro para produção de silagem de alta qualidade. Sete Lagoas: Embrapa Milho e Sorgo, 2008. 2 p. (Embrapa Milho e Sorgo. Circular Técnica, 107).

SERNA-SALDÍVAR, S. O.; CHUCKHERNÁNDEZ，C.; PÉREZ-CARRILLO, E.; EREDIA-OLEA, E. Sorghum as a Multifunctional Crop for the Production of Fuel Ethanol: Current Status and Future Trends. In: LIMA, M. A. P. (Ed.). Bioethanol. 2012. Disponível em: $<$ http:// www.intechopen.com/books/bioethanol/ sorghum-as-a-multifunctional-crop-for-theproduction-of-fuel-ethanol-current-statusand-future-trend $>$. Acesso em: 14 mai 2013. SKONIESKI, F.; NORNBERG, J.; AZEVEDO, E.; DAVID, D.; KESSLER, J.; MENEGAZ, A. Produção, caracterização nutricional e fermentativa de silagens de sorgo forrageiro e sorgo duplo propósito. Acta Scientiarum Animal Sciences, Maringá, v. 32, n. 1, 2010. Disponível em: <http://periodicos.uem. br/ojs/index.php/ActaSciAnimSci/article/ view/7200>. Acesso em: 15 dez. 2010.

SOUSA, D. M. G.; LOBATO, L. Cerrado: correção do solo e adubação. 2. ed. Brasília, DF: Embrapa Informação Tecnológica, 2004. $416 \mathrm{p}$.

UNITED NATIONS FOUNDATION. Energy 
Access Practitioner Network: Towards URQUIAGA, S.; ALVES, B. J. R.; BODDEY, R. Achieving Universal Energy Access by 2030. M.. Produção de biocombustíveis: a questão Washington: United Nations Foundation, do balanço energético. Revista de Política 2012. $60 \mathrm{p}$. Agrícola, Brasília, DF, v. 14, p. 42-46, 2005. 